\title{
Eyeball Movement Detection To Control Smart Wheelchair Using Eye Aspect Ratio (EAR)
}

\author{
Gusti Pangestu
}

\begin{abstract}
Lot of developed technology's focused on a purpose for helps the disabilities. One of them is a tools called wheelchair. Wheelchair is the most common stuff that used by disabilities as a tool for mobilization. There are two types of wheelchair. The first is the traditional wheelchair that fully operated using hand. The second is an electrical wheelchair, that assisted and operated by joystick or any electric devices. This research proposed an alternative mechanism to control the electric wheelchair by using an eyeball movement. It could be used especially for people with multiple disabilities (hand and foot defects), so they can take an advantage of their eyeballs as a tool to control wheelchair movement. There are five options for controlling the wheelchair (leftward, rightward, upward, downward and center). Leftward, rightward and center used for control direction of smart wheelchair. Furthermore, upward and downward of eye movements used to control the speed of smart wheelchair. Upward command used to increase the speed. Meanwhile, down-ward used to decrease the speed (stop). The proposed method used EAR (Eye Aspect Ratio), which divided into three regions based on sector area, for determining the directions of the eyeball movement. EAR is the value that represents the ratio between the upper eyelid and lower eyelid. The result obtained high accuracy
\end{abstract}

Index - Eye Aspect Ratio, Eye movement, Controlling

\section{INTRODUCTION}

$\mathrm{I}^{\mathrm{n}}$ recent years, disability is became a serious problem. The number of people with disability was increased so high[1]. People with disability has lot of issues, such a mobility problem, cognition problem and self-care problem. People with mobility problem has a limitation to operate their both of legs, especially for walking or something else, therefore they need a tool that can assist them to move and navigate. There is a common tool that used for disabilities person with a navigation problem called wheelchair [2]. The wheelchair is commonly used by disabilities to assist them to move and navigate using their hands by rotating the wheel to spin.

A wheelchair is a very helpful for people with navigation problem. By utilizing a wheelchair, individuals with incapacities can act and explore skirt to the typical individuals. Notwithstanding, a wheelchair is

Gusti Pangestu, is the Department of Computer Science, School of Computer Science, Bina Nusantara University (BINUS), Jakarta, Indonesia. e-mail : gusti.pangestu@binus.ac.id as yet not an answer for incapacities that have more than one inadequacy or individuals that can't work their legs and hands simultaneously. The electric wheelchair has become a solution to those problems. The utilization component of the electric wheelchair is utilizing the specific capacity of people to substitute the job of legs and hands. There are numerous specialists center around the electric wheelchair controlling strategy, for example, utilizing voice orders to control the development of the wheelchair [3], Human tracking [4], using hand gesture [5], using EEG (Electromyography) sensors [6], using biosignals [7] until use the head movement [8]. The use of those methods are not as simple as expected. As an example, using EEG sensors in human body needs an assistance to stick the electrode to the body. Also the utilization of the head movement to control the wheelchair still not practical, user or the people with multi disabilities should move their head to the left and right, and also it has a limitation because the system just only have the ability to detect left and right only. The system limitation detection generates limited capability of the wheelchair because fewer options that can be chosen. Indeed, the electric wheelchair needs another option to produce more choices and combination options.

Eye discovery is the proposed arrangement, it is a result of the comfort to identify the eye by just utilizing the camera and the capacity of the eye to move unreservedly like up, down, right, left, and ahead. There are a lot of examination zeroed in on the way to deal with identify eye development like utilizing circle location [9], but this method has limited ability to detect the movement with just only right and left gaze. Another strategy utilizing the Triangle similitude to distinguish the eyeball development, yet just can identify four look course, including right, left, up and descending and furthermore have awful exactness for recognizing the descending development about just 58\% [10]. Other research also has not good result for detecting the downward direction by using midpoint and Region Division, and also this method just can detect only four gaze direction and need a registration process too [11].

Alluding to the past issues. Our proposed strategy is to devise a way to deal with identify the 5 look course development of the eye. In this research, the eyelid also being involved to determine the direction of the eye 

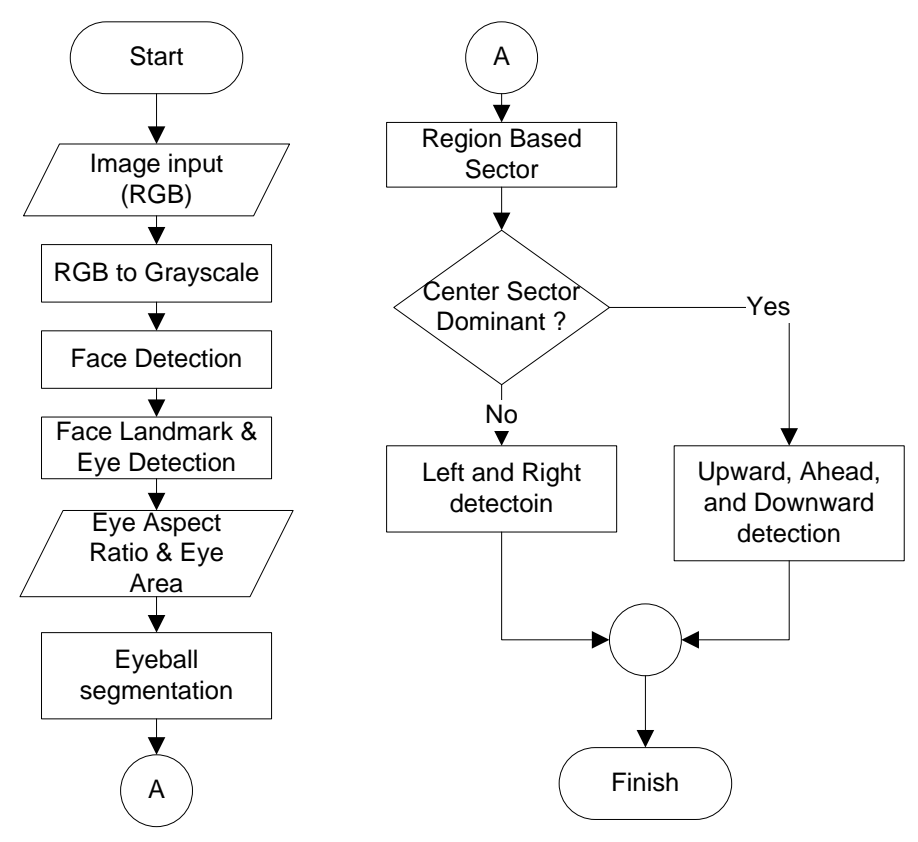

Figure 1. Eye movement direction detection schemas

movement by using EAR (Eye Aspect Ratio) [12] to detect upward and downward movement and also using three regions based eye sector to determine the right, left and ahead movement.

\section{PROPOSED METHOD}

This research combines two different approaches to produce the same output ability to detect the direction of the eye movement. These two approaches are Eye Aspect Ratio (EAR) for determining the ratio of the eyelid and 3 region based sector to determine the left, right and center direction of the eyeball movement. The detection schema also showed in the Fig. 1.

\section{A. RGB to Grayscale}

As a rule, each picture input from an external source (camera) is RGB design. In this examination, a grayscale picture is should have been handled to separate the outcome and recognize the eyeball development. Therefore, a grayscale operation is needed shown in the Eq. (1).

$$
I=\frac{R+G+B}{3}
$$

For instance, $R$ is the red value, $G$ is the green value and $B$ is the blue value in the every pixel. Afterwards, the grayscale image will be the used as the input in every process.

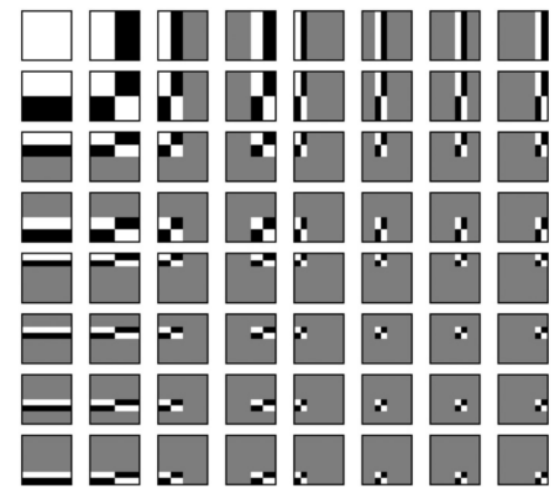

Figure 2. Haar features

\section{B. Face Detection}

Unlike the eye movement detection, eye area detection has many standard methods to apply. In this research, area of the eyes can be detected after face area can be discovered. In order to find face area, Haar Cascade became a standard method to use because of the high amount of use.

The calculation for finding or distinguishing the face region was filtered the entire whole picture utilizing the falling system [13]. Some feature (Fig. 2) composed of two or more rectangles are used for detecting the face object in the entire windows image using the Cascading process showed in the Fig. 3. The

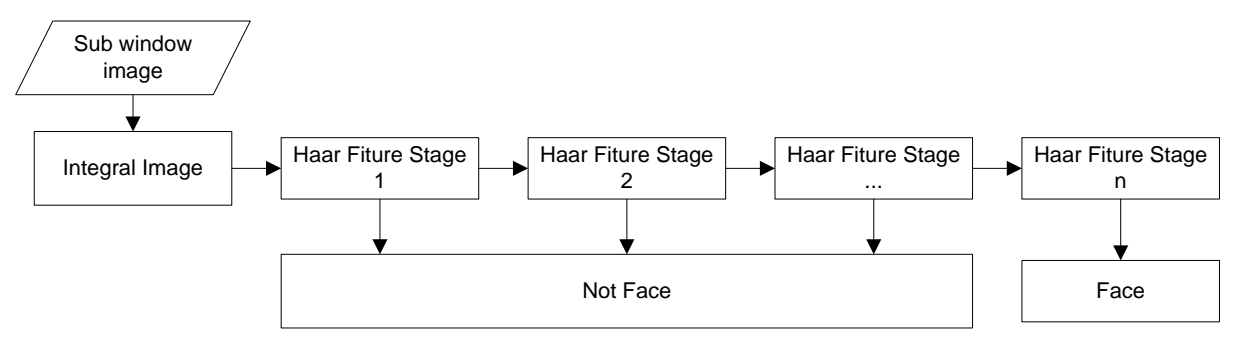

Figure 3. Haar Cascade mechanism to determine the face area 


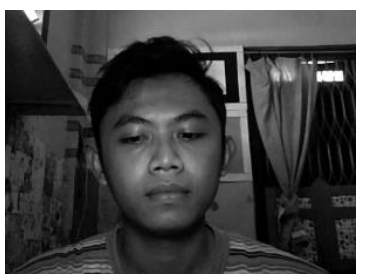

(a)

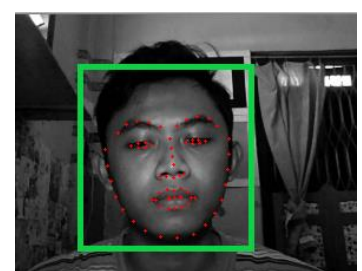

(b)
Figure 4. Haar cascade process result for detect face area, (a) image input (b) haar cascade and face landmark applied result

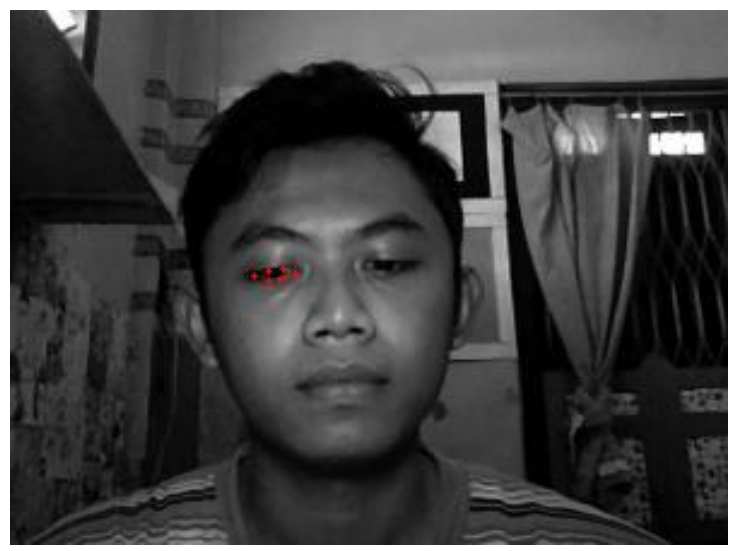

Figure 5. Eye detection result using face landmark

item can be recognized as a face in the event that it effectively passes each falling cycle. To speed up the interaction, an Integral Image utilized in this cycle. The Integral Image measure is the procedure to identify the passed on side and potential gain of the pixel to create another worth. By utilizing this new worth, the calculation cycle turns out to be quicker in light of the fact that the new worth addressing the whole picture.

A Boosting technique was likewise utilized in the Haar Cascade measure. The reason for utilizing Boosting technique is to deliver another solid student created by joining a few powerless students called Adaptive Boosting. The Adaptive Boosting equation is displayed in the Eq. (2) :

$$
h_{j}(x)=\left\{\begin{array}{c}
1 \text { if } h_{j}(x)<p_{\theta} \\
0=\text { other condition }
\end{array}\right.
$$

For instance, $h_{j}(x)$ are contain " 1 " when $h_{j}(x)$ lower than $p_{\theta}$, contain " 0 " when $h_{j}(x)$ are have another condition.

\section{Face Landmark \& Eye Detection}

The aftereffect of a Haar Cascade measure is to decide the facial regions utilized for planning the milestone in the face utilizing the Face Landmark strategy. Reffering to the research by Kazemi and Sulivan [14], a regression tree is utilized to decide the face milestone following the state of the face and coordinated with different sorts of faces information base. The result of this process are showed in the Fig. 4 marked with red dot within green rectangle.

The Eye region not really settled utilizing the Face Landmark result by taking out some superfluously MATICS: Jurnal Ilmu Komputer dan Teknologi Informasi (Journal of Computer Science and Information Technology)

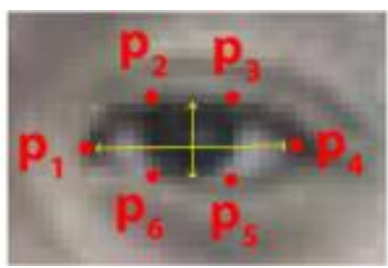

Figure 6. Dots mapping in Eye area

stamped spots in the face. In this exploration, the right eye is applied as a standard part, accordingly, every recognized face consistently identifies the right half of the eye. The aftereffect of the Eye region displayed in Fig. 5.

The EAR process utilizes the result of the eye detection process. Eye Aspect Ratio is a value to describe the distance between the upper eyelid and lower eyelid. By using the EAR process, eye shape will be determined as a value contains 0 until 1 . The value will be approaching a 0 if the upper eyelid are closest to the lower eyelid. In otherwise, the value will approaching 1 if the distance between upper eyelid and lower eyelid are move away. The EAR formulas are given in Eq. (3) :

$$
E A R=\frac{\left\|p_{2}-p_{6}\right\|+\left\|p_{3}-p_{5}\right\|}{2\left\|p_{1}-p_{4}\right\|}
$$

$P_{1}-P_{6}$ are mapping dots from the result of eye area mapping referenced in Fig. 6. Moreover, $P_{2}$ and $P_{6}$ are used as a border for top and bottom eyes, $P_{1}$ and $P_{4}$ used as a border for left and right eyes for cropping. This approach of cropping will reduce the engage of other eye pixel, so, it will reduce the calculation problem.

\section{Eyeball Segmentation}

Before adding pixel process, selected eye area must be converted to Binary image using the threshold value shown in the Eq. (4).

$$
b(i)= \begin{cases}0, & i \geq a \\ 1, & i<a\end{cases}
$$

For instance, $b$ is the binary image result, $i$ is the pixel with gray value and $a$ is the threshold value.

After the binary process was done, Morphological process applied in the binary image result to reduce the noises caused the binary process using opening showed in the Eq. (5) for the erosion step.

$$
\begin{gathered}
A \Theta B=\left\{p \in Z^{2} \mid(a+b) \in I,\right. \\
\text { for } b \in B\}
\end{gathered}
$$

$A$ is the matrix, $B$ is the kernel, if all values of 1 in $\mathrm{B}$ are contained in the position $(p)$, then $p$ is at $A \Theta B$. The erosion step is to decrease the size of the black pixel. After that, the dilation process applied by using Eq. (6).

$$
A \oplus B=\left\{z \mid\left[(\widehat{B})_{z} \cap A\right] \underline{\underline{C}} A\right\}
$$




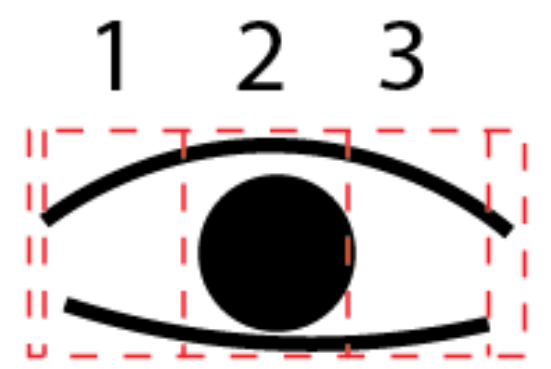

Figure 7. 3 Region Based Sectors, 1 represent the rightward, 2 represent the towards and 3 represent the leftward

Denote $A$ is the matrix and $B$ is the kernel.

\section{E. Three Region Based Sector}

Using the binary image, the next step is use the three region based sector to divides the eye area into three sectors. The sectors represent the direction of the eyeball movement shows at the Fig. 7. Three Region Based Sector process are to add all pixels in every each sector using projection method showed in Eq. (7).

$$
\text { sector }=\sum_{x=1}^{N} \sum_{y=1}^{M} I\left(b_{k}, k\right)
$$

For instance, $N$ is the vertical axis, $M$ is the horizontal axis, $b$ is the value in x coordinate, and $k$ is the value in y coordinate.

The three regions based sector process is to detect whether the eyeball moving to the rightward or to the leftward. In a case when the eyeball moved to the center or ahead, the EAR value used to determine whether eyeball moving upward, center ahead or downward. Eq. (8) shows the condition when the eyeball is looking to the center.
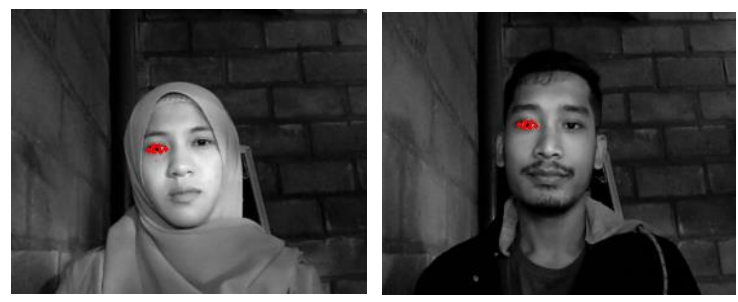

(a)

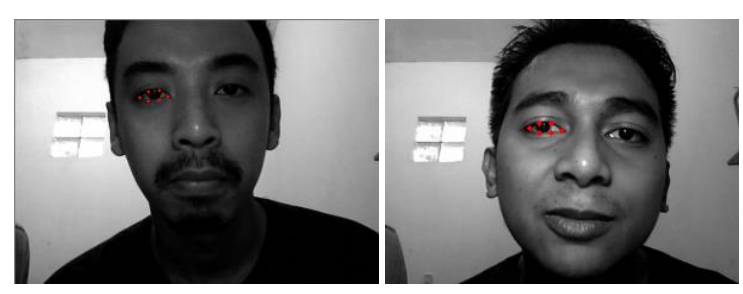

(b)

Figure 8. Eye area detection result (a) The proposed method can detect eye area with farthest distance $(40 \mathrm{~cm})$. (b) The proposed method can detect the eye area with nearest distance between face and camera $(20 \mathrm{~cm})$.
Table I. Eye area detecting experiment result

\begin{tabular}{|l|l|l|l|l|}
\hline $\begin{array}{l}\text { Distance } \\
(\mathbf{c m})\end{array}$ & Detected & $\begin{array}{l}\text { Not } \\
\text { detected }\end{array}$ & $\begin{array}{l}\text { Amount } \\
\text { of data }\end{array}$ & Accuracy \\
\hline 20 & 19 & 1 & 20 & $\mathbf{9 5 \%}$ \\
\hline 30 & 18 & 2 & 20 & $\mathbf{9 0 \%}$ \\
\hline 40 & 18 & 2 & 20 & $\mathbf{9 0 \%}$ \\
\hline
\end{tabular}

$$
d=\left\{\begin{array}{c}
\text { upward, if } E A R>t_{1} \\
\text { center, if } E A R<t_{1} \text { and } E A R \text { input }>t_{2} \\
\text { downward, if } E A R>t_{2}
\end{array}\right.
$$

Denotes, $d$ is the direction and $t$ is the average variable between center (normal) EAR to 1 (when the upper eyelid is farthest from the lower eyelid) and 0 (when the upper eyelid is closest to the lower eyelid) showed in Eq. (9) and Eq. (10).

$$
\begin{gathered}
t_{1}=\frac{E A R_{f}+0.3}{2} \\
t_{2}=\frac{E A R_{f}+0.05}{2}
\end{gathered}
$$

Denote, $E A R_{f}$ is the EAR value in the first frame when camera detects the eye area.

\section{EXPERIMENTAL RESULT}

The carried out experiment is to looking for the accuracy of the proposed method to detect the movement of the eyeball with constant lighting. First, the experiment is to find the best condition for using this proposed method include the distance and the threshold. As the information, there are total 160 amount of data that used for testing in this experiment. Those data was caputerd manualy using a 13MP camera of smartphone.

The distance variable test is required to know the ability of detecting performed by the proposed method that affected by the distance between the eyes and the camera, in this experiment the distance are 20,30, and $40 \mathrm{~cm}$ that shown in the Table I, and also this experiments using 65 as the threshold value because it produce better segmentation result based on the previous experiments.

Referring to Table I, the distance between $20 \mathrm{~cm}$ to $40 \mathrm{~cm}$ show that proposed method can detect the eye area with more than $90 \%$ average of accuracy. The sample result of eye area detecting is shown in the Fig. 8.

Table II. Result of the direction movement detection of the eyeball

\begin{tabular}{|l|l|l|l|}
\hline Direction & Correct & Not Correct & Accuracy \\
\hline Up & 18 & 2 & $90 \%$ \\
\hline Down & 17 & 3 & $85 \%$ \\
\hline Left & 18 & 2 & $90 \%$ \\
\hline Right & 18 & 2 & $90 \%$ \\
\hline Center / ahead & 18 & 2 & $90 \%$ \\
\hline
\end{tabular}


Table III. Comparison result between the proposed method and circle transform and triangle similarity approach

\begin{tabular}{|l|c|c|c|c|c|c|}
\hline \multirow{2}{*}{ Methods } & \multicolumn{5}{|c|}{ Accuracy } & Average \\
\cline { 2 - 6 } & Ahead & Right & Left & Up & Down & \\
\hline $\begin{array}{l}\text { Circle } \\
\text { Transform } \\
{[9]}\end{array}$ & $85 \%$ & $82 \%$ & $82 \%$ & - & - & $83 \%$ \\
\hline $\begin{array}{l}\text { Triangle } \\
\text { Similarity } \\
{[10]}\end{array}$ & - & $90 \%$ & $90 \%$ & $75 \%$ & $58 \%$ & $78.25 \%$ \\
\hline $\begin{array}{l}\text { Proposed } \\
\text { Method }\end{array}$ & $\mathbf{9 0 \%}$ & $\mathbf{9 0 \%}$ & $\mathbf{9 0 \%}$ & $\mathbf{9 0 \%}$ & $\mathbf{8 5 \%}$ & $\mathbf{8 9 \%}$ \\
\hline
\end{tabular}

Next experiment is to find the accuracy for detecting the direction of the eye movement using the proposed method and with random distance between the user and camera around 20 until $40 \mathrm{~cm}$. The result experiment showed in the Table II.

As shown in the Table II, the average accuracy of the proposed method is a $89 \%$ for detecting the 5 gaze including rightward, leftward, upward, downward and ahead. The sample of detection result are shown in the Fig 9.

Fig. 9 shows the result and the implementation for using eye movement detection as a wheelchair controller. In this proposed method, there are 5 gaze direction that can be detected, including upward, downward, leftward, rightward and center/ahead. By using these 5 gaze directions, a wheelchair can be controlled in the 3 different ways including rightward, leftward and center. Moreover, the speed of the wheelchair also can be controlled by upward and downward detection of eye movements a user. In this research, upward function is for speeding up the speed of the wheelchair, and the downward function is for slowing down the wheelchair.

\section{DISCUSSION}

As mentioned before, the upward and downward detection is calculated using the reference data (EAR) that saved using the first frame. Using this EAR reference data, the proposed method can determine the ratio of the eye and then compare it with frame input (real time). The graphic of EAR value when eyes looking to the center is shown in the Fig.10. The EAR value when eyes looking ahead (normal) is about 0.1 until 0.15 , when the eyeball move to the downward, the value will be changed lower as shown in the Fig. 11.

In the Fig. 11, the EAR average value when eyes look down is about 0.09 until 0.1. For example, when the first frame recorded with EAR value about 0.16 then the lower threshold $\left(t_{2}\right)$ is 0.095 . This mean, that the input EAR is lower than $t_{2}$, then eyeball is looking down. As well as the upward detection mechanism, the EAR value when eye looking upward are shown in the Fig. 12 with average result about 0.2 until 0.27 in accordance if EAR value higher than $t_{l}$, for example, when EAR reference value is 0.17 then the upper threshold $\left(t_{1}\right)$ is 0.235 , so if the EAR value input is more than 0.235 then the eyeball will be detected as upward.

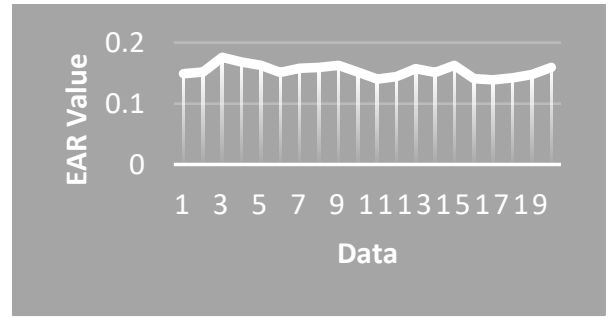

Figure 10. EAR value when eyes looking ahead / center

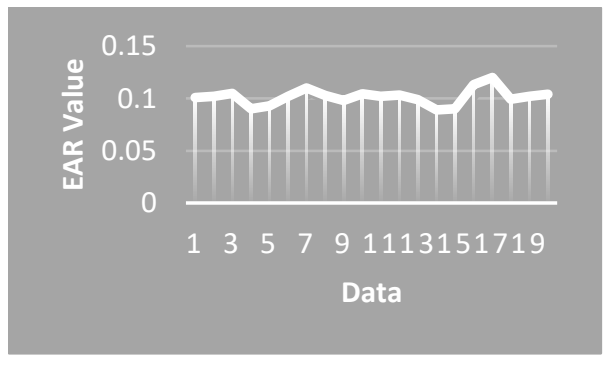

Figure 11. EAR value when eyes looking down

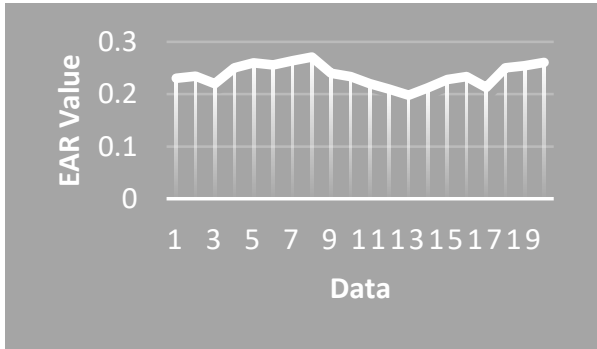

Figure 12. EAR value when eyes looking up

Our proposed method also compared with another method to find the difference. A research using the Circle Transform proposed by Utaminingrum et al [9] and other research using Triangle Similarity proposed by Reynaldi [10] shows the advancement to detect the direction of the eyeball movement. The comparison result between proposed method and another method are shown in the Table III.

Table III shows that the proposed method can outperform the other comparison method, especially the ability to detect the number of direction movements. The proposed method also can detect the 5 gaze direction, including upward, downward, leftward, rightward and ahead by $89 \%$ of accuracy. It is indicated that the proposed method also has better ability and better accuracy compared with two other methods.

\section{CONCLUSION}

In this paper, an Eye Aspect Ratio combine with region sector shows a better capability for detect the 5 gaze movements of the eyeball including upward, downward, leftward, rightward and ahead. By using the Eye Aspect Ratio (EAR) the proposed method can produce $89 \%$ of accuracy. By using eyeball movement, user with disabilities can perform and control the movement of the wheelchair by using 5 options of controlling include the speed up and slow down 


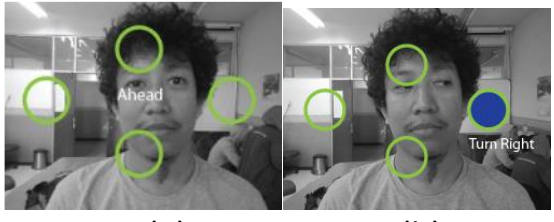

(a)

(b)

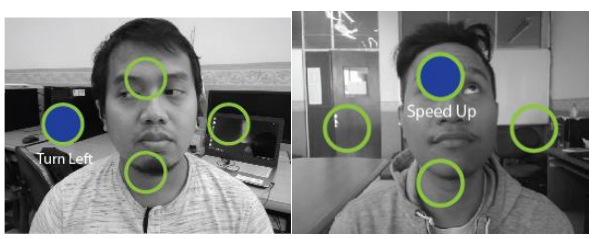

(c)

(d)

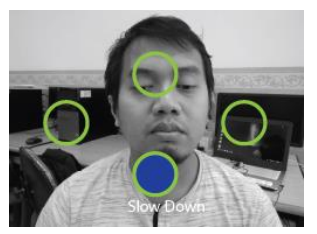

(e)

Figure 9. Eye direction detection result (a) center/ahead detection (b) rightward detection (c) leftward detection (d) upward detection (e) downward detection

movement using upward and downward eyeball movement, also leftward, ahead and rightward movement using left, right and ahead eyeball movement.

\section{REFERENCES}

[1] C. A. Okoro, N. D. Hollis, A. C. Cyrus, and S. Griffin-Blake, "Prevalence of Disabilities and Health Care Access by Disability Status and Type Among Adults - United States, 2016," MMWR. Morb. Mortal. Wkly. Rep., vol. 67, no. 32, pp. 882-887, 2018, doi:

10.15585/mmwr.mm6732a3.

[2] H. S. Jung, G. Park, Y. S. Kim, and H. S. Jung, "Development and evaluation of one-hand drivable manual wheelchair device for hemiplegic patients," Appl. Ergon., vol. 48, pp. 11-21, 2015, doi:

10.1016/j.apergo.2014.10.020.

[3] N. Peixoto, H. G. Nik, and H. Charkhkar, "Voice controlled wheelchairs: Fine control by humming," Comput. Methods Programs Biomed., vol. 112, no. 1, pp. 156-165, 2013, doi: 10.1016/j.cmpb.2013.06.009.

[4] F. Utaminingrum, T. A. Kumiawan, M. A. Fauzi, R. C. Wihandika, and P. P. Adikara, "Adaptive human tracking for smart wheelchair," 5th Int. Symp. Comput. Bus. Intell. ISCBI 2017, no. August, pp. 10-13, 2017, doi: 10.1109/ISCBI.2017.8053535.

[5] D. Orizaba, "A Hands Gesture System Of Control For An Intelligent Wheelchair.," no. 852, pp. 68-71, 2007.
[6] R. Hardiansyah, "The Electric Wheelchair Control Using Electromyography Sensor Of Arm Muscle," Int. Conf. Information, Commun. Technol. Syst., pp. 129-134, 2016.

[7] C. Ishii, S. Murooka, and M. Tajima, "Navigation of an Electric Wheelchair Using Electromyograms, Electrooculograms, and Electroencephalograms," Int. J. Mech. Eng. Robot. Res., vol. 7, no. 2, pp. 143-149, 2018, doi: 10.18178/ijmerr.7.2.143-149.

[8] F. Utaminingrum, P. P. Adikara, Y. A. Sari, D. Syauqy, and A. G. Hapsani, "Left-right head movement for controlling smart wheelchair by using centroid coordinates distance," J. Theor. Appl. Inf. Technol., vol. 96, no. 10, pp. 28522861, 2018.

[9] F. Utaminingrum, M. A. Fauzi, and Y. A. Sari, "Eye Movement as Navigator for Restricted Disabled Person in Handling Position," in ICCIS (International Conference on Communication and Information System), 2017, pp. 1-5, doi: 10.475/123.

[10] R. P. Prasetya and F. Utaminingrum, "Triangle similarity approach for detecting eyeball movement," 5th Int. Symp. Comput. Bus. Intell. ISCBI 2017, pp. 37-40, 2017, doi:

10.1109/ISCBI.2017.8053540.

[11] R. P. Prasetya, F. Utaminingrum, and W. F. Mahmudy, "Real time eyeball movement detection based on region division and midpoint position," Int. J. Intell. Eng. Syst., vol. 11, no. 3, pp. 149-158, 2018, doi: 10.22266/IJIES2018.0630.16.

[12] J. Cech and T. Soukupova, "Real-Time Eye Blink Detection using Facial Landmarks," Cent. Mach. Perception, Dep. Cybern. Fac. Electr. Eng. Czech Tech. Univ. Prague, pp. 1-8, 2016.

[13] P. Viola and M. Jones, "Rapid object detection using a boosted cascade of simple features," in Proceedings of the IEEE Computer Society Conference on Computer Vision and Pattern Recognition, 2001, vol. 1, pp. I--511, doi: 10.1109/cvpr.2001.990517.

[14] V. Kazemi and J. Sullivan, "One Millisecond Face Alignment with an Assemble of Regression Trees," Conf. Comput. Vis. Pattern Recognit., 2017, doi: 10.13140/2.1.1212.2243.

Gusti Pangestu, was born in Malang, January $5^{\text {th }}$, 1995. He graduated from Master of Computer Science (M.Kom) in 2019 from Universitas Brawijaya, Malang. Previously, he also finished his Bachelor degree in UIN Maulana Malik Ibrahim, Malang, Indonesia.

Now, he became a lecturer of Computer Science Department in Bina Nusantara University (BINUS), Jakarta, Indonesia. Previously, he also became a trainer and a software engineer in several private companies.

Based on his academic history, his full name and title also could be written with Gusti Pangestu, S.Kom., M.Kom. 